\title{
Análisis de MMPs en fluidos orales en el diagnóstico complementario de las enfermedades periodontales
}

\section{Oral-fluid MMP analysis in the complementary diagnosis of periodontal diseases}

\author{
Hernández $\mathrm{P}^{1}$, Mäntylä $\mathrm{P}^{2}$, Tervahartiala $\mathrm{T}^{3}$, Sorsa $\mathrm{T}^{4}$, Hernández $\mathrm{M}^{5}$
}

\begin{abstract}
RESUMEN
La periodontitis constituye la infección bacteriana más prevalente a nivel mundial y representa un factor de riesgo para diversas patologías sistémicas. El estado de inflamación y destrucción periodontal se manifiestan a través de la presencia de biomarcadores en el suero y fluidos orales, tales como el fluido gingival crevicular (FGC), saliva y enjuague oral. Enzimas como las metaloproteinasas de matriz (MMP) y mieloperoxidasa, constituyen biomarcadores potenciales para ensayos moleculares complementarios a la clínica de uso en el sillón dental. A continuación se presenta una revisión de la literatura respecto de la aplicación potencial del análisis de metaloproteinasas de matriz extracelular (MMPs) en el diagnóstico complementario de las enfermedades periodontales. Se ha demostrado que los niveles de MMP-9, -13 y particularmente de MMP8, se asocian con el grado de inflamación periodontal, y pueden diferenciar entre sujetos sanos, con gingivitis, periodontitis y peri-implantitis, mientras que la mejoría de los parámetros clínicos en respuesta al tratamiento periodontal se asocia con la reducción de la activación y niveles de estas enzimas en FGC, como así también en el suero. Se concluye que la determinación, particularmente de MMP-8 en fluidos orales presenta un elevado potencial como complemento de los métodos clínicos tradicionales para identificar a los pacientes con periodontitis o en riesgo de desarrollar la enfermedad, monitorear fases del tratamiento y mejoría de signos periodontales e incluso evaluar el estado de inflamación sistémica. Rev. Clin. Periodoncia Implantol. Rehabil. Oral Vol. 5(3); 150-153, 2012.
\end{abstract}

Palabras clave: MMPs, diagnóstico molecular, periodontitis, enfermedades cardiovasculares.

\section{ABSTRACT}

Periodontal disease is the most common bacterial infection worldwide and it can contribute to enhance the risk for the development of several systemic diseases. The status of periodontal inflammation and destruction can be reflected in biomarker measurement in serum and oral fluids, like gingival crevicular fluid (GCF), saliva and mouth-rinse. Some enzymes, such as matrix metalloproteinases (MMPs) and myeloperoxidase are potential candidates for chair-side point-of-care oral fluid assays. This review is focused on the utility of matrix metalloproteinase (MMP) analysis in oral fluid as a complementary diagnostic method to chronic periodontitis. Levels of MMP- $9,-13$ and specially of MMP-8, reflect oral inflammatory status and discriminate among healthy, gingivitis, periodontitis and periimplantitis individuals, whereas MMP levels and activation in GCF and serum are in line with the improvement of clinical parameters in response to periodontal treatment. As a conclusion, MMP-8 assessment in GCF could represent a helpful adjunctive method to traditional diagnostics to identify periodontitis or patients at risk to develop the disease, monitor treatment phases, improvement of periodontal signs and even screen the systemic inflammation status.

Rev. Clin. Periodoncia Implantol. Rehabil. Oral Vol. 5(3); 150-153, 2012.

Key words: MMPs, chair-side point-of-care diagnosis, periodontitis, cardiovascular diseases.

Los fluidos orales (fluido gingival crevicular (FGC), saliva, muestras de enjuagues orales y fluido sulcular peri-implantario (FSPI)) contienen mediadores moleculares llamados frecuentemente biomarcadores, los cuales son capaces de reflejar variadas condiciones fisiológicas y patológicas. Los cambios cualitativos y cuantitativos en estos biomarcadores a nivel de fluidos orales pueden reflejar el estad de salud periodontal y han demostrado ser de potencial utilidad para el diagnóstico y tratamiento de distintos desórdenes orales y sistémicos, tales como enfermedades periodontales y cardiovasculares, respectivamente ${ }^{(1)}$. Entre estos, las metaloproteinasas (MMPs) de matriz extracelular de la familia de las colagenasas, particularmente MMP8 y MMP-13, son enzimas proteolíticas que degradan los colágenos fibrilares, componentes centrales de la matriz extracelular periodontal ${ }^{(2)}$. A continuación se presenta una revisión de la literatura de los últimos 10 años respecto de la aplicación potencial del análisis de metaloproteinasas de matriz extracelular (MMPs) en el diagnóstico complementario de las enfermedades periodontales.

La periodontitis resulta de la interacción entre bacterias periodontopatógenas organizadas en la biopelícula subgingival y la respuesta inmune e inflamatoria del hospedero, y constituye la infección bacteriana más prevalente a nivel mundial. Algunos estudios revelan que $10-15 \%$ de los adultos a nivel mundial tienen periodontitis avanzada. La enfermedad periodontal puede contribuir a una disfunción severa de la salud oral, así como a un aumento de la susceptibilidad frente a otras

1. Instructor. Departamento de Odontología Conservadora. Facultad de Odontología, Universidad de Chile. Chile.

2. Prof. Asociado. Departamento de Enfermedades Orales y Maxilofaciales, Hospital Central Helsinki. Finlandia.

3. Prof. Asociado. Departamento de Enfermedades Orales y Maxilofaciales, Hospital Central Helsinki. Finlandia. Instituto de Odontología, Universidad de Helsinki. Helsinki, Finlandia.

4. Prof. Titular. Departamento de Enfermedades Orales y Maxilofaciales, Hospital Central Helsinki. Finlandia. Instituto de Odontología, Universidad de Helsinki. Helsinki, Finlandia.

5. Prof. Asistente. Departamento de Patología. Facultad de Odontología, Universidad de Chile. Laboratorio de Biología Periodontal. Facultad de Odontología, Universidad de Chile. Chile.

Correspondencia autor: Marcela Hernández Ríos. mhernandezrios@gmail.com. Financiamiento: Proyecto FIOUCH 09-10, FONDECYT 1090461 y 1120138 (MH), Fondos de la Academia de Finlandia y Hospital Central Universitario de Helsinki (TS). Trabajo recibido el 03/04/2012. Aprobado para su publicación el 21/10/2012. 
enfermedades sistémicas ${ }^{(3)}$. Adicionalmente, la infección e inflamación crónica del periodonto junto al aumento sostenido de los mediadores pro-inflamatorios pueden contribuir al desarrollo y/o progresión de patologías sistémicas, tales como diabetes, parto prematuro y bebés con bajo peso al nacer, enfermedad pulmonar, artritis y enfermedades cardiovasculares $(E C V)^{(4-6)}$. De hecho, estudios de casos y controles y de cohortes han demostrado que los pacientes con periodontitis poseen un riesgo aumentado de sufrir enfermedades cardiovasculares, infarto agudo al miocardio (IAM) y enfermedad arterial periférica en relación a los pacientes con periodonto sano ${ }^{(4-8)}$. A pesar de que se han demostrado asociaciones entre enfermedades periodontales y cardiovasculares en diversos estudios clínicos, los mecanismos patogénicos y conexiones entre ambas enfermedades aun no están completamente dilucidados ${ }^{(8,9)}$.

Las enfermedades periodontales pueden reflejarse en los fluidos orales a través de niveles elevados de enzimas proteolíticas destructivas derivadas de células y tejidos del hospedero, tales como las metaloproteinasas de matriz (MMPs) -8, -9 y -13 , elastasa de neutrófilos, $\alpha_{2}$-macroglobulina, mieloperoxidasa (MPO); mediadores proinflamatorios, como la proteína $\mathrm{C}$ reactiva (CRP), interleuquina (IL) $1 \beta$, Factor de Necrosis Tumoral (TNF) $-\alpha$, proteína inflamatoria de macrófagos (MIP)- $1 \alpha$; y marcadores de remodelación ósea, entre los cuales se encuentra la fosfatasa alcalina, fragmentos de degradación del colágeno tipo-I, ligando del receptor activador para el factor nuclear kappa beta (RANKL), osteoprotegerina y osteocalcina. Entre éstos, algunos presentan especial interés como potenciales candidatos para ensayos de análisis molecular complementario a la práctica clínica, tales como MMP-8, -9 y -13 y MPO $^{(1,2,10)}$.

En términos generales, el estado de salud periodontal se caracteriza por un bajo volumen de FGC y de los niveles de biomarcadores de destrucción tisular en todos los fluidos orales, los cuales incrementan a medida que aumenta la severidad de la inflamación periodontal(3). El análisis de biomarcadores en FGC, saliva y muestras de enjuague oral puede proveer, en conjunto con los métodos clínicos y complementarios tradicionales, información adicional a los profesionales de la salud con respecto a la presencia de la enfermedad, necesidad de tratamiento o efectividad de la medicación en la periodontitis crónica. EI FGC representa un método de análisis sitio-específico de los biomarcadores ${ }^{(11)}$. Sin embargo, el análisis molecular de este fluido puede consumir tiempo, debido a que normalmente requiere ser realizado en el laboratorio. Estos procedimientos, así como la toma de muestra de FGC, son técnicamente demandantes y el volumen de FGC obtenido puede ser escaso. A pesar de estas desventajas, el FGC aún se considera como un potencial candidato entre los fluidos orales para el desarrollo de tecnologías no invasivas de diagnóstico molecular, complementario a la clínica y de uso potencial en el sillón dental (12-17), lo cual se debe, especialmente, a que las MMPs destructoras de tejido y sus reguladores bioactivos pueden ser determinados eficazmente por distintos inmunoensayos sitio-específicos de este fluido a nivel periodontal ${ }^{(11)}$.

En comparación con el FGC, la obtención de muestras de saliva y enjuague oral también representa un método no invasivo, pero eventualmente más conveniente, práctico, rápido, y que no requiere de profesionales ni materiales especializados; incluso aún, puede ser llevado a cabo por el mismo paciente. El enjuague oral se obtiene tras administrar $1 \mathrm{~mL}$ de agua en la cavidad oral del sujeto, enjuague y colección de este $^{(10)}$. La saliva y el enjuague oral representan una muestra conjunta de todos los sitios periodontales, otorgando una evaluación general de la enfermedad periodontal o salud a nivel del sujeto. Particularmente, la saliva total puede estar afectada por constituyentes moleculares y remanentes celulares de otros nichos orales, así como por algunas condiciones sistémicas ${ }^{(18)}$ que deberían ser consideradas cuando este método es utilizado para el diagnóstico.

La MMP-8 o colagenasa-2 representa el principal tipo de colagenasa intersticial presente en FGC, FSPI, saliva, muestras de enjuague oral y tejido gingival humano afectado por periodontitis. El significado patofisiológico de un aumento de las MMPs en periodontitis depende de la interacción entre factores activadores e inhibidores endógenos, los cuales determinan la actividad de las MMPs. Todas las MMPs humanas existen en múltiples formas, tales como pro-formas latentes, formas activas, especies fragmentadas, complejos enzimáticos y formas unidas a superficie celular ${ }^{(19)}$. La expresión de diferentes isoformas de MMPs en muestras de fluidos orales puede analizarse mediante ensayos de "immunowestern blot", mientras que inmunoensayos, tales como ensayos ELISA, no logran diferenciarlas ${ }^{(9,18)}$. La determinación de las formas activas de MMPs, particularmente en relación MMPs $-8,-9$ y
-13, ha demostrado ser de gran importancia, debido a que la progresión clínica de la periodontitis y periimplantitis, tanto entre pacientes como entre sitios activos versus inactivos, se refleja fundamentalmente como una elevación patológica excesiva de las formas activas de estas MMPs en FGC, FSPI, muestras de saliva y enjuagues bucales de sitios y pacientes afectados $^{(1,2,20)}$. Las formas zimogénicas o inactivas de las MMPs -8 -9 y -13 han demostrado ser activadas por cascadas independientes y/o cooperativas que incluyen la participación de otras proteinasas del hospedero (MMPs y proteasas de serina), especies reactivas del oxígeno y mieloperoxidasa, así como también proteasas microbianas relacionadas con la progresión de la periodontitis/peri-implantitis en sitios enfermos e incluso activos ${ }^{(18,20,21)}$. La actividad de colagenasas y activación de MMP-8 en FGC también se ha asociado con los niveles de fragmentos de degradación de colágeno tipo I (ICTP). Estos reflejan la destrucción del colágeno, particularmente el del tejido óseo, superando el escudo protector provisto por el inhibidor tisular de MMPs, TIMP-1 ${ }^{(19,22)}$. La actividad de MMP-13 e ICTP han demostrado aumentar en sitios activos comparados con sitios inactivos de pacientes con periodontitis progresiva o sujetos con periodonto sano(20). Estos estudios, en conjunto, evidencian la asociación entre periodontitis crónica, progresión clínica y molecular de la enfermedad, y los niveles y/o actividad de las MMPs.

La existencia de una asociación entre la mejoría de los parámetros clínicos periodontales y la reducción de la activación y niveles de MMPs $-8,-13$ y -9 en FGC y suero se ha sostenido reiteradamente mediante ensayos clínicos que evalúan la medicación adjunta al tratamiento periodontal con dosis subantimicrobianas de doxiciclina (DSD, inhibidor sintético de MMP aprobado por la Food and Drug Administration (FDA) $)^{(1,22)}$; mientras que estudios recientes han reportado el monitoreo del efecto del tratamiento periodontal y la medicación adjunta de DSD mediante inmunoensayos de MMP-8 a través de dispositivos experimentales de uso en el sillón dental ${ }^{(1,10,11,23)}$.

Los anticuerpos para la detección de la forma activa de MMP-8 en los fluidos orales se han utilizado en ensayos de diagnóstico molecular complementario a la clínica y de uso en el sillón dental, identificando a los pacientes afectados con periodontitis y aquellos sitios susceptibles a progresión de la enfermedad(1,10,15). Recientemente, nuestro grupo de investigación determinó los niveles de MMP-8 en FGC con dos tests de uso en clínica diferentes (dentoAnalyzer, por dentognostics $\mathrm{GmbH}$ y el test de de varilla, específico para MMP-8); y dos métodos cuantitativos de laboratorio (ensayo inmunofluorométrico (IFMA), y ELISA comercial)(11). dentoAnalyzer es un test clínico que determina valores cuantitativos para el marcador en estudio, mientras que el test de varilla presenta resultados dicotómicos respecto de un valor de corte a partir de una muestra de fluido oral. Los resultados de IFMA, dentoAnalyzer y el test de varilla específicos para MMP-8 fueron concordantes. Contrariamente, los resultados obtenidos mediante ELISA comercial para MMP-8 no estuvieron en línea con los otros métodos. Tanto IFMA como dentoAnalyzer determinaron los niveles de MMP. 8 en las muestras de FGC con igual confiabilidad. Los resultados del test de varilla fueron concordantes con los resultados de estos otros dos métodos, pero la sensibilidad fue menor y sólo detectó los sitios con niveles elevados de MMP-8. Adicionalmente, los sitios inestables es decir aquellos que sufrieron pérdida de inserción tras el tratamiento periodontal, demostraron niveles repetidamente elevados de MMP-8 determinados mediante IFMA y dentoAnalyzer (Figuras 1 y 2$)^{(11)}$.

Las diferencias entre dentoAnalyzer, IFMA, test de varilla y ELISA comercial para el análisis de niveles de MMP-8 en el FGC pueden ser explicadas, al menos en parte, por las evidentes diferencias entre las especificidades y sensibilidades de los anticuerpos utilizados en estos ensayos. DentoAnalyzer, IFMA y el test de varilla se basan en el uso del mismo anticuerpo ${ }^{(24)}$. El anticuerpo utilizado en dentoAnalyzer, IFMA y test de varilla presenta una alta sensibilidad tanto para los isotipos de MMP-8 de neutrófilos como los de fibroblastos, y especialmente hacia sus formas activas ${ }^{(24,25)}$. Si bien la evidencia disponible no es concluyente, ambos métodos presentan resultados promisorios.

Sobre la base de estos antecedentes, los inmunoensayos de MMP-8 y ensayos de niveles de actividad para especies de isoenzimas de MMP-8 de neutrófilos en fluidos orales, podrían resultar especialmente útiles en la diferenciación de pacientes o sitios con periodontitis y periimplantitis, gingivitis, e incluso sujetos sanos ${ }^{(1,2,11,15,16)}$. Si bien el examen clínico periodontal es necesario y no puede ser reemplazado en el diagnóstico periodontal, un test para un biomarcador específico o un conjunto de ellos podría otorgar información adjunta relevante sobre los niveles de respuesta individual del hospedero, a pesar de que aún no 

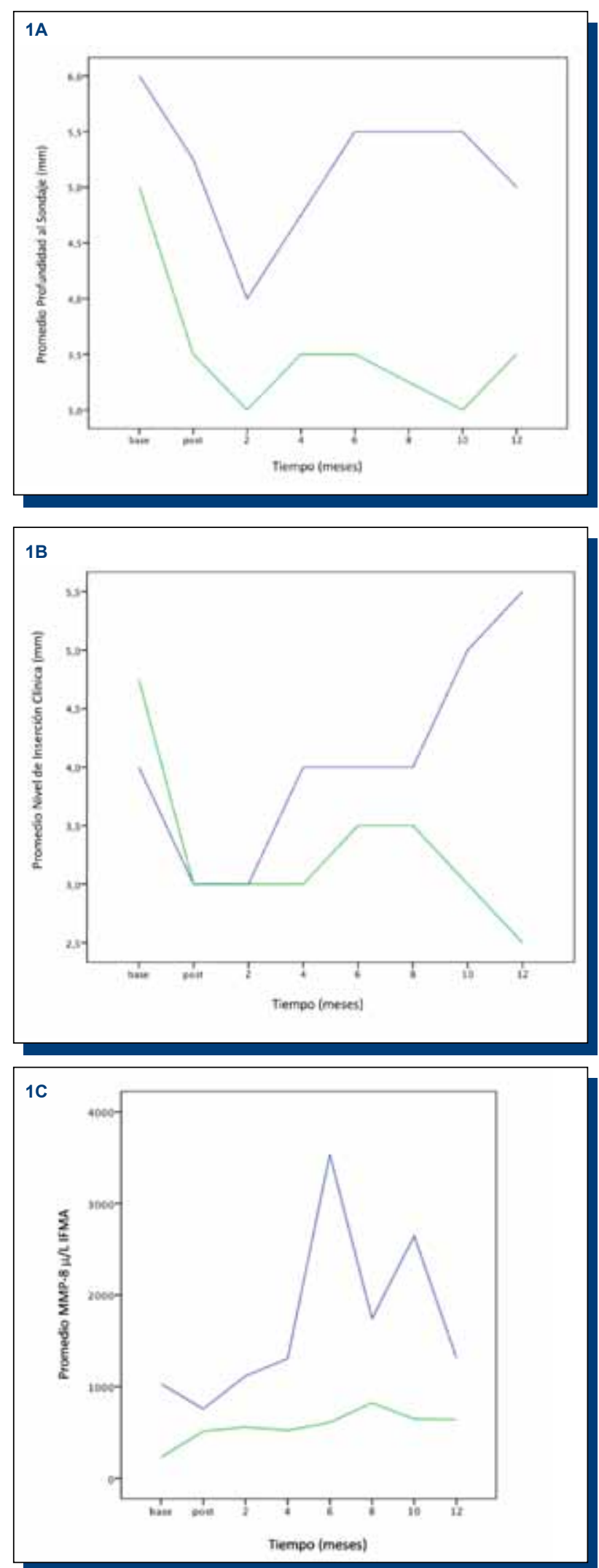

Figura 1. Curva de sitios inestables (línea azul) y sitios estables (línea verde), pertenecientes a nueve pacientes fumadores con periodontitis ( $n=14$ y 65 , respectivamente). Promedio de (A) Profundidad al sondaje; (B) Nivel de inserción clínica; y (C) Niveles de MMP-8 en fluido gingival crevicular en el línea base (base), un mes posterior al tratamiento (post), y en las visitas de mantención desde los 2 hasta los 12 meses.

se han establecido los valores considerados realmente "normales". Si bien diversos estudios han demostrado el rol central de MMP-8 en la patogenia de la periodontitis y como potencial biomarcador, aún no se
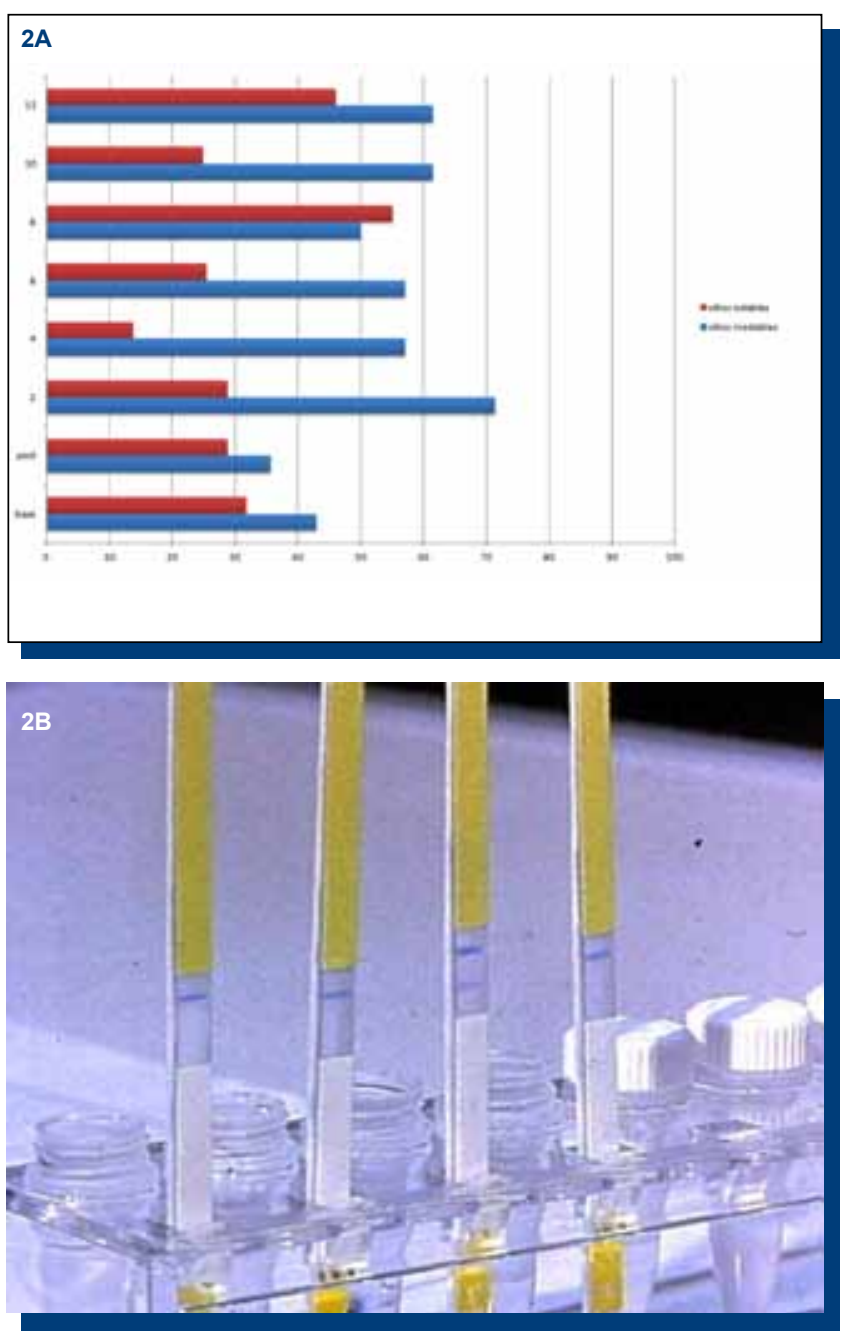

Figura 2. (A) Resultados positivos de MMP-8 determinados por Test de Varilla en sitios estables e inestables de 9 sujetos con periodontitis. Resultados expresados como \%; Punto de corte $1000 \mu \mathrm{g} / \mathrm{L}$, para los mismo sitios y tiempos de la Figura 1.

(B) Test de Varilla específico para MMP-8 (segundo a la izquierda). El resultado positivo es confirmado mediante la aparición de una línea azul en la zona de captura.

ha demostrado su rol predictivo en la progresión de la enfermedad ${ }^{(26)}$. Este problema surge debido a la naturaleza de la periodontitis y la falta de precisión en el diagnóstico de sitios activos mediante los métodos clínicos o radiológicos. La progresión de la periodontitis crónica es infrecuente, episódica y lenta ${ }^{(15,27)}$, afecta a un número reducido de sitios, y sólo un pequeño grupo de pacientes con periodontitis demuestran múltiples sitios activos ${ }^{(28)}$. Sin embargo, se puede concluir que niveles repetidamente elevados de MMP-8 en FGC señalan los sitios en riesgo de sufrir pérdida de inserción periodontal y que la medición de MMP-8 en el sitio, específicamente en FGC, es una ayuda diagnóstica valiosa que complementa los métodos tradicionales, específicamente en sitios de pacientes periodontales en la fase de mantención que están en riesgo continuo de recurrencia de la enfermedad. Los niveles de MMP-8 en FGC de pacientes periodontales disminuyen después de la fase higiénica del tratamiento convencional. Sin embargo, los niveles permanecen más altos que en los pacientes con gingivitis o que en el FGC de pacientes sanos $^{(16)}$. Esto es válido también en sitios poco profundos de pacientes con periodontitis y en sitios sin pérdida de inserción, y reflejaría la respuesta basal elevada del hospedero en los sujetos con periodontitis ${ }^{(15,16)}$.

Según hallazgos recientes ${ }^{(1,10)}$, los análisis de MMP-8 con ensayos complementarios en muestras salivales y enjuagues bucales pueden ser clínicamente útiles para realizar una identificación general de los individuos con periodontitis o para analizar el nivel individual de respuesta del hospedero. Los análisis simultáneos de MMP-8 y TIMP-1 también podrían resultar beneficiosos ${ }^{(10)}$. Las pruebas en base a fluidos orales podrían otorgar información adicional valiosa sobre el control de la inflamación, la cual hoy en día se basa principalmente en hallazgos clínicos como la disminución de la profundidad de sacos periodontales y disminución del sangrado al sondaje. El efecto inhibitorio de MMP-8 por 
medicaciones de DSD podría ser monitoreado a través del análisis de niveles de MMP-8 salival y en muestras de enjuague oral, de modo de identificar cuándo detener la administración de la medicación, o cuándo la medicación debería ser administrada nuevamente ${ }^{(22,29)}$. Estos ensayos en muestras de saliva o enjuague oral también podrían ser aplicables para la diferenciación entre gingivitis y periodontitis, debido a que los niveles de MMP-8 de pacientes con gingivitis han demostrado ser significativamente más bajos que en los pacientes con periodontitis ${ }^{(16)}$. Así, los niveles que se aproximen repetidamente a los encontrados en periodontitis podrían ser utilizados para identificar a los pacientes en riesgo.

Adicionalmente, las inmuno-tecnologías de diagnóstico molecular complementarias a la clínica y de uso en el sillón dental, tanto aquellas de análisis de fluidos orales como las de aplicación en suero, bien podrían ser adaptadas para monitorear incluso la inflamación sistémica ${ }^{(18,30)}$. La elevación de MMP-8 tanto en suero como plasma se ha asociado con infarto agudo del miocardio (IAM), enfermedades cardiovasculares $(E C V)$ y sepsis ${ }^{(1,18,31}$. Además, un estudio reciente de MMP-8 salival medida por análisis de "immunowestern blot" en pacientes con y sin infarto agudo al miocardio reveló que la activación elevada de MMP-8 salival está asociada con infarto al miocardio(18). Por otra parte, un estudio multicéntrico de cohorte prospectivo demostró recientemente que los niveles séricos de MMP-8 eran significativamente mayores entre los pacientes con sepsis no sobrevivientes que entre los sobrevivientes.
Así, niveles elevados de MMP-8 en fluidos orales y suero pueden ser considerados como un potencial factor de riesgo para enfermedades sistémicas tales como, pero no únicamente, ECV, sepsis, diabetes, infarto, artritis y enfermedades pulmonares. En adición, la activación oral de MMP-8 como consecuencia de IAM puede contribuir con una mayor progresión de la periodontitis y de otras condiciones bucales. Sin embargo, el diagnóstico molecular a través de fluidos orales puede contener varios potenciales confundentes. Las múltiples medicaciones, en conjunto con la presencia de enfermedades sistémicas (desórdenes mentales, enfermedades psiquiátricas, síndrome nefrótico) pueden afectar los niveles de biomarcadores en periodontitis y ECV, tanto en saliva como en suero o plasma ${ }^{(1,4,18)}$.

Como conclusión general, los ensayos moleculares complementarios a la clínica de uso en el sillón dental basados en MMP-8 poseen un enorme potencial para el diagnóstico oral, y más aún, pueden representar un eslabón entre la inflamación oral y condiciones inflamatorias sistémicas.

\section{CONFLICTOS DE INTERÉS}

Los autores declaran no tener conflictos de interés.

\section{REFERENCIAS BIBLIOGRÁFICAS}

1. Sorsa T, Tervahartiala T, Leppilahti J, Hernández M, Gamonal J, Tuomainen AM et al. Collagenase-2 (MMP-8) as a point-of-care biomarker in periodontitis and cardiovascular diseases. Therapeutic response to non-antimicrobial properties of tetracyclines. Pharmacol Res, 2011; 63(2): 108-113.

2. Hernández M, Gamonal J, Tervahartiala T, Mantyla P, Rivera O, Dezerega A et al. Associations between matrix metalloproteinase- 8 and -14 and myeloperoxidase in gingival crevicular fluid from subjects with progressive chronic periodontitis: A longitudinal study. J Periodontol, 2010; 81(11): 1644-1652.

3. Pussinen PJ, Paju S, Mantyla P, Sorsa T. Serum microbial- and host-derived markers of periodontal diseases: A review. Curr Med Chem, 2007; 14(22): 24022412.

4. Alfakry H, Paju S, Sinisalo J, Nieminen MS, Valtonen V, Saikku P et al. Periodontopathogen - and host-derived immune response in acute coronary syndrome. Scand J Immunol, 2011 Oct; 74(4): 383-389.

5. Chen C, Nan B, Lin P, Yao Q. C-reactive protein increases plasminogen activator inhibitor-1 expression in human endothelial cells. Thromb Res, 2008; 122(1): 125 133.

6. Mattila KJ Pussinen PJ, Paju S. Dental infections and cardiovascular diseases: A review. J Periodontol, 2005; 76(11 Suppl): 2085-2088.

7. Persson RE, Hollender LG, MacEntee MI, Wyatt CC, Kiyak HA, Persson GR. Assessment of periodontal conditions and systemic disease in older subjects. J Clin Periodontol, 2003; 30(3): 207-213.

8. Bahekar AA, Singh S, Saha S, Molnar J, Arora R. The prevalence and incidence of coronary heart disease is significantly increased in periodontitis: A meta-analysis. Am Heart J, 2007; 154(5): 830-837.

9. Buduneli N, Kinane DF. Host-derived diagnostic markers related to soft tissue destruction and bone degradation in periodontitis. J Clin Periodontol, 2011; 38 Suppl 11: 85-105.

10. Leppilahti J, Ahonen MM, Hernandez M, Munjal S, Netuschil L, Uitto VJ et al. Oral rinse MMP-8 point-of-care immuno test identifies patients with strong periodontal inflammatory burden. Oral Dis, 2011 Jan; 17(1): 115-122.

11. Sorsa T, Hernández M, Leppilahti J, Munjal S, Netuschil L, Mantyla P. Detection of gingival crevicular fluid MMP-8 levels with different laboratory and chair-side methods. Oral Dis, 2010; 16(1): 39-45.

12. Sorsa $T$, Ding $Y$, Salo $T$, Lauhio A, Teronen $O$, Ingman $T$ et al. Effects of tetracyclines on neutrophil, gingival, and salivary collagenases. A functional and western-blot assessment with special reference to their cellular sources in periodontal diseases. Annals of the New York Academy of Sciences, 1994; 732: 112-131.

13. Sorsa $T$, Tjaderhane $L$, Konttinen $Y T$, Lauhio A, Salo $T$, Lee $H M$ et al. Matrix metalloproteinases: Contribution to pathogenesis, diagnosis and treatment of periodontal inflammation. Ann Med, 2006; 38(5): 306-321.

14. Sorsa T, Tjaderhane L, Salo T. Matrix metalloproteinases (MMPs) in ora diseases. Oral Dis, 2004; 10(6): 311-318.

15. Mantyla P, Stenman M, Kinane D, Salo T, Suomalainen K, Tikanoja S et al. Monitoring periodontal disease status in smokers and nonsmokers using a gingival crevicular fluid matrix metalloproteinase-8-specific chair-side test. J Periodontal Res, 2006; 41(6): 503-512.

16. Mantyla P, Stenman M, Kinane DF, Tikanoja S, Luoto H, Salo T et al. Gingival crevicular fluid collagenase-2 (MMP-8) test stick for chair-side monitoring of periodontitis. J Periodontal Res, 2003; 38(4): 436-439.
17. Munjal SK, Prescher N, Struck F, Sorsa T, Maier K, Netuschil L. Evaluation of immunoassay-based MMP-8 detection in gingival crevicular fluid on a point-of-care platform. Annals of the New York Academy of Sciences, 2007; 1098: 490-492.

18. Buduneli E, Mantyla P, Emingil G, Tervahartiala T, Pussinen P, Baris N et al Acute myocardial infarction is reflected in salivary matrix metalloproteinase- 8 activation level. J Periodontol, 2011; 82(5): 716-725.

19. Sorsa T, Mantyla P, Tervahartiala T, Pussinen PJ, Gamonal J, Hernández M MMP activation in diagnostics of periodontitis and systemic inflammation. $J$ Clin Periodontol, 2011: 38(9): 817-819.

20. Hernández Ríos M, Sorsa T, Obregon F, Tervahartiala T, Valenzuela MA, Pozo P et al. Proteolytic roles of matrix metalloproteinase (MMP)-13 during progression of chronic periodontitis: Initial evidence for MMP-13/MMP-9 activation cascade. J Clin Periodontol, 2009; 36(12): 1011-1017.

21. Hernández M, Dutzan N, Garcia-Sesnich J, Abusleme L, Dezerega A, Silva N et al. Host-pathogen interactions in progressive chronic periodontitis. J Dent Res, 2011; 90(10): 1164-1170

22. Reinhardt RA, Stoner JA, Golub LM, Lee HM, Nummikoski PV, Sorsa T et al. Association of gingival crevicular fluid biomarkers during periodontal maintenance with subsequent progressive periodontitis. J Periodontol, 2010; 81(2): 251-259.

23. Gursoy M, Kononen E, Gursoy UK, Tervahartiala T, Pajukanta R, Sorsa T. Periodontal status and neutrophilic enzyme levels in gingival crevicular fluid during pregnancy and postpartum. J Periodontol, 2010; 81(12): 1790-1796.

24. Hanemaaijer R, Sorsa T, Konttinen YT, Ding Y, Sutinen M, Visser $\mathrm{H}$ et al. Matrix metalloproteinase- 8 is expressed in rheumatoid synovial fibroblasts and endothelial cells. Regulation by tumor necrosis factor-alpha and doxycycline. $\mathrm{J}$ Biol Chem 1997; 272(50): 31504-31509.

25. Sorsa $T$, Mantyla $P$, Ronka $H$, Kallio $P$, Kallis GB, Lundqvist $C$ et al Scientific basis of a matrix metalloproteinase-8 specific chair-side test for monitoring periodontal and peri-implant health and disease. Annals of the New York Academy of Sciences, 1999; 878: 130-140.

26. Masada MP, Persson R, Kenney JS, Lee SW, Page RC, Allison AC Measurement of interleukin-1 alpha and -1 beta in gingival crevicular fluid: Implications for the pathogenesis of periodontal disease. J Periodontal Res, 1990; 25(3): 156-163. 27. Chambers DA, Imrey PB, Cohen RL, Crawford JM, Alves ME, McSwiggin TA. A longitudinal study of aspartate aminotransferase in human gingival crevicular fluid. $J$ Periodontal Res, 1991; 26(2): 65-74.

28. Hernández M, Valenzuela MA, López-Otin C, Álvarez J, López JM, Vernal R et al. Matrix metalloproteinase-13 is highly expressed in destructive periodontal disease activity. J Periodontol, 2006; 77(11): 1863-1870.

29. Golub LM, Lee HM, Stoner JA, Sorsa T, Reinhardt RA, Wolff MS et al Subantimicrobial-dose doxycycline modulates gingival crevicular fluid biomarkers of periodontitis in postmenopausal osteopenic women. J Periodontol, 2008; 79(8): 1409-1418.

30. Tuomainen AM, Nyyssonen K, Laukkanen JA, Tervahartiala T, Tuomainen TP, Salonen JT et al. Serum matrix metalloproteinase-8 concentrations are associated with cardiovascular outcome in men. Arterioscler Thromb Vasc Biol, 2007; 27(12): 2722-2728.

31. Lauhio A, Hastbacka J, Pettila V, Tervahartiala T, Karlsson S, Varpula T et al. Serum MMP-8, -9 and TIMP-1 in sepsis: High serum levels of MMP-8 and TIMP1 are associated with fatal outcome in a multicentre, prospective cohort study. Hypothetical impact of tetracyclines. Pharmacol Res, 2011 Dec; 64(6): 590-594. 